
\title{
28 Research Square \\ Relationship between structural and functional changes in glaucomatous eyes: A multifocal electroretinogram study
}

Hiroki Tanaka

Gifu Daigaku Igakubu Daigakuin Igakukei Kenkyuka

Kyoko Ishida ( $\nabla$ kyoko.ish@gmail.com )

Toho Daigaku Iryo Center Ohashi Byoin https://orcid.org/0000-0001-5141-1000

Kenji Ozawa

Gifu Daigaku Igakubu Daigakuin Igakukei Kenkyuka

Takuma Ishihara

Gifu University

Akira Sawada

Gifu Daigaku

Kiyofumi Mochizuki

Gifu Daigaku Igakubu Daigakuin Igakukei Kenkyuka

Tetsuya Yamamoto

Gifu Daigaku Igakubu Daigakuin Igakukei Kenkyuka

\section{Research article}

Keywords: multifocal electroretinography, optical coherence tomography, Humphrey Field Analyzer, open angle glaucoma

Posted Date: October 2nd, 2020

DOI: https://doi.org/10.21203/rs.2.20694/v2

License: (c) (1) This work is licensed under a Creative Commons Attribution 4.0 International License.

Read Full License

Version of Record: A version of this preprint was published at BMC Ophthalmology on August 21 st, 2021. See the published version at https://doi.org/10.1186/s12886-021-02061-8. 


\section{Abstract}

Background: The nasal to temporal amplitudes ratio (N/T) of multifocal electroretinography (mfERG) scans measured within $5^{\circ}$ of the macula can be used to detect glaucomatous change. The photopic negative response (PhNR) of $\mathrm{mfERG}$ elicited by a circular stimulus centered on the fovea was significantly reduced in eyes with glaucoma. The PhNR to B-wave ratio $(\mathrm{PhNR} / \mathrm{B})$ is the optimal measure of the PhNR. However, clinical superiority for evaluating glaucoma patients has not been determined between N/T and PhNR/B yet. Methods: For morphological assessments, ganglion cell complex (GCC) in six regions and the average were measured by optical coherence tomography (OCT). For functional assessment, Humphrey visual fields (VF) with mean sensitivities (MT) and mfERG scans with parameters of N/T and the multifocal photopic negative response to B-wave ratio ( $\mathrm{mfPhNR} / \mathrm{B}$ ) were measured. Sixtynine eyes of forty-four glaucoma patients were included and correlations between mfERG parameters and OCT or VF parameters were evaluated. Results: The mean age of patients was 59.4 years. The mean deviation for all eyes obtained with the VF 30-2 and VF 10-2 was -7.00 and $-6.31 \mathrm{~dB}$, respectively. Significant correlations between GCC thickness or VF parameter and the N/T were found, especially in the inferior and inforotemporal retinal areas corresponding to superior and superonasal VF sectors (GCC vs $\mathrm{N} / \mathrm{T}$; coefficient $=-7.916$ and -7.857 , and MT vs N/T ; coefficient $=-4.302$ and -4.437 , in the inferior and inforotemporal retinal areas, respectively, all $p$ values $<0.05$ ). However, similar associations were not obtained between mfPhNR/B and OCT or VF parameters. The mfPhNR/B only in the inferotemporal sector was significantly correlated with the average thickness of GCC (coefficient $=4.823, P=0.012$ ). Conclusions: The N/T is better parameter than PhNR/B for the assessment of glaucomatous eyes in the current study's measurement conditions. A future study modifying the stimuli and amplitudes to obtain the spatial correspondence to OCT and VF measurement will be required to evaluate the value of mfERG.

\section{Background}

Glaucomatous optic neuropathy is associated with the loss of retinal ganglion cells (RGCs) and their axons. The photopic negative response (PhNR) of the full-field electroretinography (ERG) is a slow negative potential following the $a$ - and b-waves that has been reported to originate primarily from the neural activities of the RGCs [1-3]. The amplitudes of the PhNR in focal ERG scans were significantly reduced in eyes with glaucoma [3-6]. However, the PhNR can be measured in several different ways, either as the negative trough following the b-wave or at a fixed time point [7-9]. Wu et al. recently reported that the PhNR to B-wave ratio (PhNR/B; B-wave amplitude defined as the a-wave trough to bwave peak) exhibited the lowest magnitude of test-retest variability and concluded that PhNR/B was the optimal measure of the PhNR [10].

In earlier reports, the nasal to temporal amplitudes ratio (N/T) of the first slice of the second-order kernels of multifocal electroretinography (mfERG) scans measured within $5^{\circ}$ of the macula was smaller in glaucoma patients than in normal subjects. Further, a significant correlation was present between N/T and visual field (VF) parameters or the retinal thickness in the inferior quadrant in eyes with moderate glaucoma $[11,12]$. 
These findings suggest that $\mathrm{PhNR} / \mathrm{B}$ and N/T might be helpful in determining functional defects in patients with glaucoma and in diagnosing glaucoma. However, clinical superiority for evaluating glaucoma patients has not been determined between PhNR/B and N/T yet.

There are several reports available outlining direct comparisons among visual sensitivities determined by standard automated perimetry (SAP), structural parameters of the inner retina obtained by optical coherence tomography (OCT), and the amplitude of the PhNR/B or the N/T [4, 5, 13-15], but no reports especially conducting comparisons between $\mathrm{N} / \mathrm{T}$ and the PhNR/B of mfERG scans (mfPhNR/B) in the same glaucoma patient have been published.

Thus, the aims of this study are to investigate the association between the morphological statuses of the macular region by OCT, the functional status including two mfERG parameters with N/T and $\mathrm{mfRhNR/B}$, and the sensitivities of SAP and to determine the clinical superiority between $\mathrm{mfPhNR/B}$ and $\mathrm{N} / \mathrm{T}$ in the same glaucoma patients.

\section{Materials And Methods}

\subsection{Subjects}

This was an observational cross-sectional study of patients treated at the Glaucoma Service of the Gifu University Hospital over a six-year period. We obtained written informed consent from all participants and all of the procedures conformed to the tenets of the Declaration of Helsinki. The Institutional Board of Research Associates of Gifu University Graduate School of Medicine approved our research protocols.

Open angle glaucoma (OAG) diagnoses were based on the presence of normal open-angle and glaucomatous optic nerve changes corresponding to VF defects. We classified the patients as having normal tension glaucoma (NTG) if none of the recorded intraocular pressures (IOPs) exceeded $21 \mathrm{mmHg}$ in either eye at all examinations, while the remaining patients were classified as having primary OAG (POAG). Patients eligible for study inclusion had clinical diagnoses of POAG or NTG, a refractive spherical equivalent ranging between -6.0 diopters (D) and $+3.0 \mathrm{D}$, and a best-corrected visual acuity (VA) of 0 logarithm of the minimum angle of resolution (logMAR) units or less.

We excluded patients with intraocular abnormalities other than glaucoma; those with significant cataracts that could induce refractive or VF errors; those with a history of any medication use that could affect the pupillary diameter, those with intraocular surgeries including laser therapy; and those with medical treatment changes in the interval among the VF tests, OCT examinations, and mfERG recordings. All examinations including VF, OCT, and mfERG were performed each other within six months. When both eyes met the criteria, two eyes of the patient were included in the study.

\section{2. $O C T$}


Pupils were dilated with topical $0.5 \%$ tropicamide and $0.5 \%$ phenylephrine (Mydrin- ${ }^{\circledR}$; Santen Pharmaceutical, Osaka, Japan) before the OCT examinations with a Cirrus high-definition OCT (HD-OCT) 4000 instrument (Carl Zeiss Meditec, Jena, Germany). The software automatically collected measurements of the peripapillary RNFL with a diameter of $3.46 \mathrm{~mm}$ consisting of 256 A-scans centered on the optic disc. We obtained the average thickness of the circumpapillary RNFL (cpRNFL), then used the Macula Cube $200 \times 200$ and Ganglion Cell Analysis (GCA) programs to collect additional data in glaucoma patients as follows.

The macular cube scan generated one set of 200 horizontal B-scans, each composed of 200 A-scans centered on a 6- $\times$ 6-mm macular region. The built-in GCA algorithm (Cirrus H-OCT software, version 6.0) measured the thicknesses of the macular RNFL (mRNFL) and ganglion cell-inner plexiform layer (GCIPL) within a 6- $\times 6-\times 2-\mathrm{mm}$ cube in an elliptical annulus around the fovea. By using the GCA algorithm, the GCIPL thickness was calculated automatically as the distance from the outer boundary of the RNFL to the outer boundary of the inner plexiform layer (IPL) and stratified such as global and sectoral values (i.e., superonasal, superior, superotemporal, inferotemporal, inferior, and inferonasal sectors). All of the sectorial thickness obtained with OCT were shown based on the corresponding VF sectors. (Figure 1a and 1b). We also measured the mRNFL thickness as the distance between the internal limiting membrane and the outer boundary of the RNFL and calculated the same six sectorial values. Ganglion cell complex (GCC) was measured as the value which added MRNFL with GCIPL and we also calculated the six sectorial values of GCC, similarly.

We only incorporated OCT images with a high quality of signal strength greater than $7 / 10$ in the analysis.

\subsection{VF testing}

All glaucoma participants underwent perimetric examinations using the Humphrey Field Analyzer (HFA) (750 I series; Carl Zeiss Meditec, Jena, Germany) with the Central 30-2 (HFA 30-2) and the Central 10-2 programs (HFA 10-2) using the Swedish Interactive Threshold Algorithm. We identified glaucomatous VF defects by the presence of three or more significant $(P<0.05)$ non-edge-contiguous points, with at least one point located at the $P<0.01$ level in the pattern deviation plot along with grading outside the normal limits in the glaucoma hemifield test. VF tests were considered reliable when false-negative responses were less than $15 \%$, false-positive responses were less than $15 \%$ and fixation losses were less than $20 \%$. Based on the report of RGC displacement, we classified the stimulus points on the HFA 10-2 corresponding to the six sectors of the GCIPL measurement ellipse into six groups (Figure 1b) [16]. We averaged the thresholds of each sector on the SAP.

\section{4. $m f E R G$ scans}

All glaucoma patients underwent mfERG. We used the Visual Evoked Response Imaging System Science (VERIS) 5.1.10× (Electro-Diagnostic Imaging, Milpitas, CA, USA) to record mERG scans according to a published method $[11,12,14]$. After pupils were dilated to at least $8 \mathrm{~mm}$ in diameter with Mydrin- $\mathrm{P}^{\circledR}$, we placed a bipolar contact lens electrode (Mayo, Inazawa, Japan) on the anesthetized (oxybuprocaine 
hydrochloride, Benoxil ${ }^{\circledR}$; Santen Pharmaceutical, Osaka, Japan) cornea. We covered the contralateral eye, and then applied hydroxyethylcellulose gel (Scopisol ${ }^{\circledR}$; Senju Pharmaceutical, Osaka, Japan) to the cornea to protect it from dehydration and to achieve good electrical contact between the electrodes and the cornea. We attached a gold-cup electrode to the right earlobe as a ground electrode. We then carried out refractions to elucidate the patients' best VA for the stimulus viewing distance. Next, we adjusted the viewing distance to compensate for changes in the retinal image size due to the refractive lens used. During the mfERG recordings, the subjects sat with their chin and forehead tightly fixed. We instructed the subjects to fixate on a point at the center of the cathode ray tube (CRT) monitor while the eyes were being stimulated. The distance from the tested eye to the CRT monitor was $33 \mathrm{~cm}$ at zero diopters. The amplitudes of the mfERG were expressed as the response density, $\mathrm{nV} / \mathrm{deg}^{2}$, or $\mu \mathrm{V}$, representing the amplitudes as a function of the stimulus area.

\subsection{P1 Component of the first slice of second-order kernels of mfERG scans}

The visual stimuli consisted of 37 hexagons that were displayed on a monochrome computer monitor (QB1781; Chuomusen, Tokyo, Japan) (Figure 2a). The stimulus array subtended a visual angle of $50^{\circ}$ by $40^{\circ}$. Each hexagonal element of the stimulus was independently alternated between black $\left(5 \mathrm{~cd} / \mathrm{m}^{2}\right)$ and white (200 cd/ $\mathrm{m}^{2}$; contrast: $\left.95.1 \%\right)$ at a frame rate of $75 \mathrm{~Hz}$ according to a binary m-sequence. We set the bandpass filters at 10 to $300 \mathrm{~Hz}$. We monitored the positions of the eyes during the recordings through the VERIS recording window. Each recording lasted approximately four minutes, and we discarded segments with eye movements or blinking artifacts and recorded them again. We applied an artifact elimination technique once, with no spatial smoothing [17]. We studied the amplitudes of the first positive peak, P1 (Figure 2b). The P1 amplitudes of the first slice of the second-order kernel responses were measured according to a published method $[11,12,14]$.

\subsubsection{Nasal to temporal amplitude ratio analyses of $\mathrm{mfERG}$ scans}

The mfERG scans elicited by the 37-hexagon stimulus array within a circle of a $5^{\circ}$ radius are shown in Figure 2a. We compared the summed mfERG scans from the central $5^{\circ}$ of the nasal VF (i.e., temporal hemisphere of the central $5^{\circ}$ retinal area; red color in Figures $2 \mathrm{a}$ and $2 \mathrm{c}$ ) with those in the temporal hemisphere of the central $5^{\circ} \mathrm{VF}$ (i.e., nasal hemisphere of the central $5^{\circ}$ retinal area; orange color in Figures 2a and 2c). We calculated the N/T-namely, the ratio of the mfERG P1 amplitudes of the first slice

of the second-order kernel (Figure 2b) -in the nasal hemisphere of the VF (i.e., temporal hemisphere of the retina) and compared with that in the temporal hemisphere of the VF (i.e., nasal hemisphere of the retina) in the central $5^{\circ}$. We also calculated the correlations between the thresholds obtained from the corresponding VF area, OCT parameters, and the N/T $[18,19]$.

\subsubsection{Multifocal photopic negative response}

The mfPhNRs were elicited by a circular stimulus with a $5^{\circ}$ radius centered on the fovea and by a quarter of an annulus placed in the superotemporal, superonasal, inferotemporal, and inferonasal regions around 
the fovea (Figure 3a). The radius of the inner border of the annulus was $5^{\circ}$ and that of the outer border was $20^{\circ}$. White $\left(200 \mathrm{~cd} / \mathrm{m}^{2}\right)$ or black $\left(5 \mathrm{~cd} / \mathrm{m}^{2}\right)$ elements were presented in a pseudorandom binary $\mathrm{m}$ sequence at a frequency of $37.5 \mathrm{~Hz}$. Each recording lasted approximately two minutes. A steady background surrounded the stimulus field. We measured the multifocal a-wave amplitude from the baseline to the trough of the first negative response and the multifocal B-wave (mfB-wave; P1-N1) from the first negative trough to the peak of the following positive wave [20]. The PhNR was measured from the baseline to the negative trough at more than $70 \mathrm{~ms}$ from the stimulus onset (Figure 3b) [6].

\subsection{4. $m f P h N R / B$ analyses}

We calculated the amplitudes of the mfPhNR/B in each sector. To compare the mfPhNR/B with the corresponding VF findings, we measured the thresholds with the HFA 30-2 and averaged for the same sectors according to the distance from the macula within the central $20^{\circ}$ (Figure 4). We also calculated the correlations between the thresholds of the corresponding VF area, the $\mathrm{mfPhNR} / \mathrm{B}$, and OCT parameters $[18,19]$.

\subsection{Statistical analyses}

The demographic data of glaucoma patients was summarized using mean $\pm S D$ with range for continuous variables and frequencies for categorical variables. To assess the relationship between parameters, we used the multivariable regression model with Huber-White robust sandwich estimator because the data encompassed repeated observations (right and left eyes) in the same patient. The multivariable regression model was adjusted for covariates including age and spherical equivalent as potential confounder. Moreover, we used the restricted cubic splines to allow for nonlinear associations between parameters. Because nonlinearity was taken into account in the parameters, the coefficients for changes from the 25th percentile to the 75th percentile were reported as representative. A two-sided significance level was 0.05 . We accepted an association only if the p-value of the statistical test of the regression coefficient was below the significance level. All analyses were performed using R software (www.rproject.org).

\section{Results}

\subsection{Demographic data of glaucoma patients}

The demographics of the 69 eyes of 44 patients with OAG included in this study are presented in Table 1. The mean patient age was 59.4 years, while the mean IOP for all eyes was $13.8 \mathrm{mmHg}$. Seventeen eyes had POAG and 52 had NTG. Thirty-two eyes were using topical antiglaucoma medications. The mean deviation (MD) for all eyes obtained with the HFA 30-2 was -7.00 dB and the pattern standard deviation (PSD) was $9.01 \mathrm{~dB}$. Similarly, the MD with the HFA 10-2 was $-6.31 \mathrm{~dB}$ and the average PSD was $7.85 \mathrm{~dB}$. Forty eyes were early, 13 were middle-stage, and 16 were advanced glaucoma. The mean cPRNFL, GCIPL, mRNFL, and GCC was $69.8,68.9,26.7$, and $95.6 \mu \mathrm{m}$, respectively. 
3.2. Correlation between the $N / T$ of $M f E R G$, the macula thickness of OCT, and the mean thresholds and the total deviation of HFA 10-2 in each sector in glaucoma patients

The N/T was significantly correlated with the average thickness of GCIPL (coefficient $=-3.455, P=0.009$ ) (Table 2). The N/T was also significantly correlated with the thickness of GCIPL in superotemporal, superior, superonasal, and inferotemporal sectors, which corresponded to inferonasal, inferior, inferotemporal, and superonasal sectors of the retina (coefficient $=-4.767, P=0.003$, coefficient $=-3.461$, $P=0.014$, coefficient $=-5.311, P=0.001$, and coefficient $=-3.027, P=0.032$, respectively) .

For mRNFL, the N/T was significantly correlated with the average thickness of mRNFL (coefficient = -2.354, $P=0.034$ ), and with the thickness of mRNFL in superotemporal, superior, and superonasal sectors , which corresponded to inferonasal, inferior, and inferotemporal sectors of the retina (coefficient $=-3.665$, $\mathrm{P}=0.026$, coefficient $=-4.455, \mathrm{P}=0.008$, coefficient $=-2.546, \mathrm{P}=0.007$, respectively) .

For GCC, the N/T was significantly correlated with the average thickness of GCC (coefficient $=-5.809, P=$ 0.017), and with the thickness of GCC in superotemporal, superior, and superonasal sectors, which corresponded to inferonasal, inferior, and inferotemporal sectors of the retina (coefficient $=-8.432, \mathrm{P}=$ 0.007 , coefficient $=-7.916, P=0.008$, coefficient $=-7.857, P=0.001$, respectively) .

For VF, the N/T was significantly correlated with MT in superior, superonasal, and inferotemporal sectors (coefficient $=-4.302, P=0.045$, coefficient $=-4.437, P=0.020$, and coefficient $=-0.864, P=0.026$, respectively). The $\mathrm{N} / \mathrm{T}$ was also significantly correlated with TD in superonasal sector (coefficient $=$ $-4.823, \mathrm{P}=0.018)$.

3.3. The $m f P h N R / B$ of $m f E R G$, the mean threshold and total deviation of HFA 30-2, and the correlations between them in each sector in glaucoma patients

The $\mathrm{mfPhNR} / \mathrm{B}$ was significantly negatively correlated with the MT and the total deviation in the superotemporal sector (coefficient $=-1.632, P=0.013$, and coefficient $=-1.701, P=0.018$, respectively) (Table 3).

3.4. Correlations between the $m f P h N R / B$ of $m f E R G$ in each sector and the average thickness of the GCIPL, $m R N F L$, and GCC of OCT in glaucoma patients

The $\mathrm{mfPhNR} / \mathrm{B}$ in the superotemporal and inferotemporal sector was significantly correlated with the average thickness of GCIPL (coefficient $=-0.025, P=0.033$ and coefficient $=2.459, P=0.042$, respectively) (Table 4$)$.

The $\mathrm{mfPhNR} / \mathrm{B}$ in the inferotemporal sector was significantly correlated with the average thickness of $\mathrm{mRNFL}$, and GCC (coefficient $=2.363, \mathrm{P}=0.003$ and coefficient $=4.823, \mathrm{P}=0.012$, respectively).

\section{Discussion}


This study investigated the association between the morphological statuses of the macular region measured by OCT, the functional status including two mfERG parameters with $\mathrm{N} / \mathrm{T}$ and $\mathrm{mfRhNR/B}$, and the sensitivities of SAP and determined the clinical superiority between mfPhNR/B and N/T in the same glaucoma patients. Better correlations between VF parameters or OCT thickness and the N/T were found, comparing with the $\mathrm{mfRhNR/B}$.

In this study, the P1 component of the first slice of the second-order kernel response was elicited by the stimuli in the central $5^{\circ}$ region in OAG patients (Figure 2). However, we adopted the N/T instead of the P1 component because the latter boasts relatively large intersubject variations [12]. The nasal-temporal asymmetry of the mfERG scans is affected in glaucomatous eyes [11,12]. We previously reported significant differences existed in the N/T of the first slice of the second-order kernel of the $\mathrm{mfERG}$ scans in the central $5^{\circ}$ between normal and NTG eyes and found significant correlations between the N/T and the MT obtained with the HFA $30-2$ and 10-2 results $[11,14]$, which are in agreement with the current study in glaucoma patients. The N/T was significantly correlated with the MT of HFA 10-2 in the superior and the superonasal sectors and TD in superonasal sector (Table 2). In the current study, we used not only the N/T of mfERG scans and HFA 10-2 but also OCT parameters and, furthermore, we classified the stimulus points on the HFA 10-2 as corresponding to each of six GCIPL measurement ellipse sectors into six groups (Figure 1) based on the report of RGC displacement [16]. Although GCC thickness can predict function within the central area in eyes with glaucoma, adjusting for the RGC displacements is essential in evaluating the association between structure and function in the macula [21]. Resultantly, both relationships-between N/T and mRNFL, GCIPL, and GCC thickness-had significant correlations in the superior, superotemporal, and superonasal areas (i.e. inferior, inferonasal, and inferotemporal retina areas) (Table 2). Furthermore, the GCIPL thickness in the inferotemporal sector (i.e. superonasal retina area) significantly correlated with N/T. Correction coefficients of GCIPL and GCC thickness to N/T were higher than those of mRNFL thickness to N/T. Since N/T of mfERG is derived from RGC, GPIPL including RGC cell bodies and their dendrites or GCC including RGC cell bodies, their dendrites, and their axons might show better associations to N/T than mRNFL axons. We also found statistically significant correlations exist between the MT and N/T (Table 2) in the superior and superonasal VF sectors (i.e., inferior and inferotemporal retinal areas). These findings may be related to unique glaucomatous $\mathrm{VF}$ defect patterns found in the superior and superonasal areas that correspond to inferior and inferotemporal retinal damages [16]. Lee et al. reported that progressive GCIPL thinning in the temporal sector occurred faster in affected than in unaffected hemifields [16]. Na et al. found that the macula cube volume and the thicknesses of the temporal and inferior macular sectors decreased faster in progressively glaucomatous eyes [22]. These reports are in agreement with our findings.

The nasal amplitudes in the first slice of the second-order kernel of mfERG within $5^{\circ}$ were significantly smaller than the temporal amplitudes in normal subjects $[11,12]$ (The average $N / T$ ratio in the normal subjects was 0.64 with the mean age of 61.9 years [11]), whereas the difference became smaller and thus the N/T ratio became larger (approaching 1.0) after glaucoma development and progression. If the difference in amplitude became insignificant (i.e., saturated) in a very early stage of glaucoma, the ratio reached 1.0 in the early stage of glaucoma progression and, thus, the N/T was not useful clinically for 
monitoring glaucomatous functional change; however, the nasal amplitudes were still significantly smaller than the temporal amplitudes $\left(3.05 \mathrm{nV} / \mathrm{deg}^{2}\right.$ in the nasal vs. 3.92 in the temporal hemifields; $P<$ $0.001, \mathrm{~N} / \mathrm{T}=0.81$ ) in this study population with an average MD of $-7.00 \mathrm{~dB}$, i.e., patients with moderate glaucoma. Thus, these abovementioned studies and ours suggest that glaucomatous changes are often found in the superior or superonasal regions of central VFs and that N/T of mfERG in the central $5^{\circ}$ may be useful for detecting glaucomatous VF and the corresponding inferior or inferotemporal inner OCT changes at least until reaching the moderate stage of disease.

The PhNR amplitudes of the focal macular ERG scans can be used to assess the damages of the RGCs in glaucoma and the decrease in the PhNR amplitudes was associated with reductions in the cPRNFL and mRNFL thicknesses $[6,13]$. The amplitudes of the PhNR of the focal ERG scans correlated with the corresponding $\mathrm{cpRNFL}$ thicknesses when measured by scanning laser polarimetry in the superotemporal and inferotemporal regions [23]. Recently, the PhNR/B was reported to exhibit the lowest magnitude of test-retest variability and to be the optimal measure of the PhNR [10]. In the current study, we measured the $\mathrm{mfPhNR} / \mathrm{B}$. Among sectorial values of the $\mathrm{mfPhNR} / \mathrm{B}$, significant correlation was found between the inferotemporal region of $\mathrm{mfPhNR/B}$ and the average GCIPL, $\mathrm{mRNFL}$ and GCC thickness (Table 4). The $\mathrm{mfPhNR} / \mathrm{B}$ in the supeotemporal was significantly negatively correlated with GCIPL (Table 4), and the MT, and total deviation in the superotemporal VF area (i.e. inferonasal retinal area) (Table 3). The decrease in the PhNR/B amplitudes should be associated with reductions in the retinal thickness and sensitivities. One of the reasons for this discrepancy may be regional disagreement in the measurement areas. Better association (The coefficient of GCC was the highest) was observed between the full GCC and PhNR/B, comparing to RNFL or GCIPL. The comparison between the mfPhNR/B and OCT macular parameters is limited by the difference in spatial correspondence- the main OCT parameters cover a much larger region than the central stimuli, and a much smaller region than the stimuli in the outer annulus. Thus, better association was observed to use the full GCC rather than MRNFL and MGCIPL parameters separately, as this is generally more robust given the low resolution of the HD-OCT Macular Cube scan. Machide et al. also found that the PhNRs of focal ERG were well-correlated with the GCC thickness within the central macula [5]. Kaneko et al. used mfERG to assess the PhNR recorded from five macular retinal locations and found selective reductions in the mfERG component only present within the central 15 degrees. Thus, another possibility is that the PhNR/B may be most useful within the central macula because of the highest RGC density being in the macula [15]. A multifocal technique could assess multiple independent stimulus locations simultaneously; however, the best way to go about topographic analysis has not yet been elucidated [24]. Further research that the measurement areas of PhNR/B correspond to those of OCT to elucidate the utility of the PhNR/B.

In our study, correlations between VF parameters or OCT thickness and the N/T were found, especially in the inferior and inforotemporal retinal areas corresponding to superior and superonasal VF sectors. Initial glaucomatous changes tend to occur in these areas [16, 22]. However, similar results were not obtained with between mfPhNR/B and VF or OCT parameters. In the current study's measurement conditions, the $\mathrm{N} / \mathrm{T}$ is better parameter than $\mathrm{mfPhNR} / \mathrm{B}$ in the assessment of glaucomatous eyes. A future study 
modifying the stimuli and amplitudes in the central and four more peripheral area to obtain the spatial correspondence to OCT measurement will be required to evaluate the value of $\mathrm{mfPhNR/B}$. OCT measurement is a fast and completely noninvasive test. VF measurement is affected by the subject's voluntarily. For patients whose VF tests are not reliable, only OCT measurement is the tool for objective assessment in glaucoma. The best way to go about topographic analysis of mfERG parameters has not yet been proven and further study is required regarding whether mfERG parameters in conjunction with OCT measurements in the corresponding macular region may enhance diagnostic sensitivity in glaucoma. For another subjective assessment tool of OCT, mfERG will be supplemental ones in the future if further improvements are achieved.

\section{Conclusions}

The $\mathrm{N} / \mathrm{T}$ is better parameter than $\mathrm{mfPhNR} / \mathrm{B}$ for the assessment of glaucomatous eyes in the current study. A future study modifying the stimuli and amplitudes to obtain the spatial correspondence to OCT and VF measurement will be required to evaluate the value of mfERG.

\section{Abbreviations}

mfERG: Multifocal electroretinography

MT:Mean thresholds

VF: Visual field

mRNFL: Macular retinal nerve fiber layer

GCIPL: Ganglion cell and inner plexiform layer

OCT: Optical coherence tomography

N/T: Nasal to temporal amplitudes ratio

$m f P h N R / B$ : Multifocal photopic negative response to B-wave ratio

RGCs: Retinal ganglion cells

PhNR: Photopic negative response

PhNR/B: photopic negative response to B-wave ratio

ERG: Electroretinography

SAP:Standard automated perimetry

OAG: Open angle glaucoma 
NTG: Normal tension glaucoma

IOPs: Intraocular pressures

POAG: Primary open-angle glaucoma

D: Diopters

$V A$ :Visual acuity

IogMAR: Logarithm of the minimum angle of resolution

RNFL: Retinal nerve fiber layer

HD-OCT: High-definition optical coherence tomography

cpRNFL: Circumpapillary retinal nerve fiber layer

GCA: Ganglion Cell Analysis

IPL: Inner plexiform layer

GCC: Gganglion cell complex

HFA: Humphrey Field Analyzer

HFA 30-2: Humphrey Field Analyzer with the Central 30-2 programs

HFA 10-2: Humphrey Field Analyzer with the Central 10-2 programs

VERIS: Visual Evoked Response Imaging System Science

CRT: Cathode ray tube

mfB-wave: Multifocal B-wave

MD: Mean deviation

PSD: Pattern standard deviation

\section{Declarations}

Ethics approval and consent to participate: The study followed the tenets of the Declaration of Helsinki and was approved by the Institutional Board of Research Associates of Gifu University Graduate School of Medicine. A written consent for participation was taken from each patient before the measurement.

Consent for publication: Not applicable 
Availability of data and materials: The data used in the current study is available from the corresponding author upon request.

Competing interests: The authors declare that they have no competing interests

Funding: No funding was received for this research.

Authors contributions: $\mathrm{KI}$ and $\mathrm{KM}$ have made substantial contributions to conception and design, and interpretation of data; $\mathrm{HT}, \mathrm{KO}$ and $\mathrm{AS}$ have made a substantial contribution to acquisition of data; $\mathrm{HT}, \mathrm{KI}$ and KM have been involved in drafting the manuscript; TY and TI provided critical revision; $\mathrm{HT}, \mathrm{KI}$ and TI performed the statistical analyses. All authors read and approved the final manuscript.

Acknowledgements

We would like to thank all of the participants involved in this study.

\section{References}

1. Viswanathan S, Frishman LJ, Robson JG, Harwerth RS, Smith EL $3^{\text {rd }}$. The photopic negative response of the macaque electroretinogram: reduction by experimental glaucoma. Invest Ophthalmol Vis Sci. 1999;40(6):1124-1136.

2. Drasdo N, Aldebasi YH, Chiti Z, Mortlock KE, Morgan JE, North The s-cone PHNR and pattern ERG in primary open angle glaucoma. Invest Ophthalmol Vis Sci. 2001;42(6):1266-1272.

3. Machida S, Tamada K, Oikawa T, Gotoh Y, Nishimura T, Kaneko M, Kurosaka D. Comparison of photopic negative response of full-field and focal electroretinograms in detecting glaucomatous eyes. J Ophthalmol. 2011;2011.pii:564131.

4. Falsini B, Marangoni D, Salgarello T, Stifano G, Montrone L, Campagna F, Alibert S,Balestrazzi E, Colotto A. Structure-function relationship in ocular hypertension and glaucoma: interindividual and interocular analysis by OCT and pattern ERG. Graefes Arch Clin Exp Ophthalmol. 2008;246(8):11531162.

5. Machida S, Kaneko M, Kurosaka D. Regional variations in correlation between photopic negative response of focal electoretinograms and ganglion cell complex in glaucoma. Curr Eye Res. 2015;40(4):439-449.

6. Machida S, Tamada K, Oikawa T, Yokoyama D, Kaneko M, Kurosaka D. Sensitivity and specificity of photopic negative response of focal electoretinogram to detect glaucomatous eyes. $\mathrm{Br} \mathrm{J}$ Ophthalmol. 2010;94(2):202-208.

7. Viswanathan S, Frishman LJ, Robson JG, Walters JW. The photopic negative response of the flash electroretinogram in primary open angle glaucoma. Invest Ophthalmol Vis Sci. 2001;42(2):514-522. 
8. Wang J, Cheng H, Hu YS, Tang RA, Frishman LJ. The photopic negative response of the flash electroretinogram in multiple sclerosis. Invest Ophthalmol Vis Sci. 2012;53(3):1315-1323.

9. Tang J, Edwards T, Crowston JG, Sarossy M. The Test-Retest Reliability of the Photopic Negative Response (PhNR). Transl Vis Sci Technol. 2014;3(6):1. eCollection 2.

10. Wu Z, Hadoux X, Hui F, Sarossy MG, Crowston JG. Photopic Negative Response Obtained Using a Handheld Electroretinogram Device: Determining the Optimal Measure and Repeatability. TransI Vis Sci Technol. 2016;5(4):8. eCollection

11. Asano E, Mochizuki K, Sawada A, Nagasaka E, Kondo Y, Yamamoto T. Decreased nasal-temporal asymmetry of the second-order kernel response of multifocal electroretinograms in eyes with normaltension glaucoma. Jpn J Ophthalmol. 2007;51(5):379-389.

12. Takagi S, Tomita G, Nagasaka E, Eguchi S. Second-order component of multifocal electroretinogram in eyes with glaucoma. Rinsho Ganka (Jpn J Clin Ophthalmol). 2014;68:1427-1432.

13. Cvenkel B, Sustar M, Perovšek D. Ganglion cell loss in early glaucoma, as assessed by photopic negative response, pattern electroretinogram, and spectral-domain optical coherence tomography. Doc Ophthalmol. 2017;135(1):17-28.

14. Hori N, Komori S, Yamada H, Sawada A, Nomura Y, Mochizuki K, Yamamoto T. Assessment of macular function of glaucomatous eyes by multifocal electroretinograms. Doc Ophthalmol. 2012;125(3):235-247.

15. Kaneko M, Machida S, Hoshi Y, Kurosaka D. Alterations of photopic negative response of multifocal electroretinogram in patients with glaucoma. Curr Eye Res. 2015;40(1):77-86.

16. Lee WJ, Kim YK, Park KH, Jeoung JW. Trend-based Analysis of Ganglion Cell-Inner Plexiform Layer Thickness Changes on Optical Coherence Tomography in Glaucoma Progression. Ophthalmology. 2017;124(9):1383-1391.

17. Sutter EE and Tran D. The field topography of ERG components in man. 1. The photopic luminance response. Vision Res. 1992;32(3):433-446.

18. Brandao LM, Ledolter AA, Monhart M, Schötzau A, Palmowski-Wolfe AM. Ganglion cell layer segmentation and the two-flash multifocal electroretinogram improve structure function analysis in early glaucoma. Graefes Arch Clin Exp Ophthalmol. 2017 Oct;255(10):1991-2000.

19. Brandao LM, Monhart M, Schötzau A, Ledolter AA, Palmowski-Wolfe AM. Wavelet decomposition analysis in the two-flash multifocal ERG in early glaucoma: a comparison to ganglion cell analysis and visual field. Doc Ophthalmol. 2017;135(1):29-42.

20. Van Astine AW, Viswanathan S. Test-retest reliability of the mutifocal photopic negative response. Doc Ophthalmol. 2017;134(1):25-36.

21. Ohkubo S, Higashide T, Udagawa S, Sugiyama K, Hangai M, Yoshimura N, Mayama C, Tomidokoro A, Araie $\mathrm{M}$, Iwase $\mathrm{A}$, et al. Focal relationship between structure and function within the central 10 degrees in glaucoma. Invest Ophthalmol Vis Sci. 2014;55(8):5269-5277.

22. Na JH, Sung KR, Lee JR, Lee KS, Baek S, Kim HK, Sohn YH. Detection of glaucomatous progression by spectral-domain optical coherence tomography. Ophthalmology. 2013;120(7):1388-1395. 
23. Tamada K, Machida S, Oikawa T, Miyamoto H, Nishimura T, Kurosaka D. Correlation between photopic negative response of focal electroretinograms and local loss of retinal neurons in glaucoma. Curr Eye Res. 2010;35(2):155-164.

24. Wilsey LJ, Fortune B. Electroretinography in glaucoma diagnosis. Curr Opin Ophthalmol. 2016r;27(2):118-124

\section{Tables}

Table 1 The Demographic data of glaucoma patients 


\begin{tabular}{|c|c|c|}
\hline \multicolumn{2}{|l|}{ Variable } & $\mathrm{N}=44$ \\
\hline \multicolumn{2}{|l|}{ Gender (male/female) } & 22 eyes/ 22 eyes \\
\hline \multicolumn{2}{|l|}{ Eye (Right/Left) } & 32 eyes/ 37 eyes \\
\hline \multicolumn{2}{|l|}{ Age [years] } & $59.4 \pm 11.8(35 \sim 78)$ \\
\hline \multicolumn{2}{|c|}{ Type of Glaucoma (POAG/NTG) } & 17eyes/ 52eyes \\
\hline \multicolumn{2}{|c|}{ Corrected Visual acuity (Log MAR) } & $-0.13 \pm 0.06(-0.18 \sim 0.00)$ \\
\hline \multicolumn{2}{|c|}{ Spherical Equivalent [Diopters] } & $-1.94 \pm 2.35(-5.88 \sim+3.00)$ \\
\hline \multicolumn{2}{|c|}{ Intraocular Pressure [mmHg] } & $13.8 \pm 2.8(7 \sim 21)$ \\
\hline \multicolumn{2}{|l|}{ Medication (With/Without) } & $32 / 37$ \\
\hline \multirow[t]{5}{*}{$\mathrm{mfPhNR} / \mathrm{B}$} & Center & $0.32 \pm 0.10(0.14 \sim 0.65)$ \\
\hline & Superotemporal & $0.21 \pm 0.09(0.07 \sim 0.69)$ \\
\hline & Superonasal & $0.21 \pm 0.06(0.10 \sim 0.41)$ \\
\hline & Inferonasal & $0.25 \pm 0.07(0.12 \sim 0.42)$ \\
\hline & Inferotemporal & $0.25 \pm 0.07(0.12 \sim 0.47)$ \\
\hline \multicolumn{2}{|l|}{$\mathrm{N} / \mathrm{T}$} & $0.83 \pm 0.37(0.11 \sim 2.50)$ \\
\hline \multicolumn{3}{|c|}{ HFA Central 30-2 Program } \\
\hline \multicolumn{2}{|l|}{ Mean Deviation $[\mathrm{dB}]$} & $-7.00 \pm 7.34(-29.92 \sim 2.06)$ \\
\hline \multicolumn{2}{|c|}{ 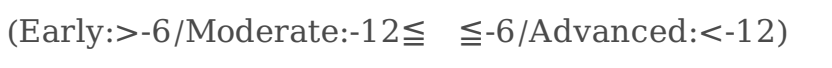 } & $40 / 13 / 16$ \\
\hline \multicolumn{2}{|c|}{ Pattern Standard Deviation [dB] } & $9.01 \pm 5.23(1.61 \sim 18.76)$ \\
\hline \multirow[t]{2}{*}{ Mean Threshold $[\mathrm{dB}] /$} & Center & $27.1 \pm 7.0(9.8 \sim 35.5) /-6.0 \pm 6.8(-25.3 \sim 2.3)$ \\
\hline & Superotemporal & $19.8 \pm 8.8(2.2 \sim 32.4) /-8.0 \pm 9.2(-29.9 \sim 2.8)$ \\
\hline \multirow{3}{*}{ Total Deviation } & Superonasal & $21.1 \pm 11.6(0.0 \sim 33.1) /-9.3 \pm 12.1(-32.0 \sim 2.9)$ \\
\hline & Inferonasal & $22.6 \pm 10.4(0.0 \sim 33.4) /-8.7 \pm 10.9(-33.1 \sim 2.8)$ \\
\hline & Inferotemporal & $23.8 \pm 6.3(0.0 \sim 30.6) /-4.3 \pm 7.1(-32.6 \sim 2.0)$ \\
\hline \multicolumn{3}{|l|}{ HFA Central 10-2 Program } \\
\hline \multicolumn{2}{|l|}{ Mean Deviation [dB] } & $-6.31 \pm 6.91(-27.42 \sim 2.07)$ \\
\hline \multicolumn{2}{|c|}{ Pattern Standard Deviation [dB] } & $7.85 \pm 5.66(0.98 \sim 17.19)$ \\
\hline \multirow[t]{2}{*}{ Mean Threshold $[\mathrm{dB}] /$} & Superotemporal & $29.5 \pm 7.4(0 \sim 37.2) /-2.7 \pm 6.1(-31.8 \sim 2.6)$ \\
\hline & Superior & $22.9 \pm 13.3(0 \sim 36.8) /-10.0 \pm 13.6(-35.5 \sim 3.0)$ \\
\hline \multirow{4}{*}{ Total Deviation } & Superonasal & $25.1 \pm 12.1(0.2 \sim 36.8) /-9.5 \pm 12.6(-35.6 \sim 3.2)$ \\
\hline & Inferonasal & $30.9 \pm 6.8(5.42 \sim 37.2) /-3.3 \pm 7.0(-29.6 \sim 3.2)$ \\
\hline & Inferior & $31.1 \pm 6.0(6.5 \sim 36.5) /-2.1 \pm 5.7(-28.3 \sim 2.0)$ \\
\hline & Inferotemporal & $32.5 \pm 4.0(10.2 \sim 36.2) /-0.4 \pm 2.1(-7.0 \sim 3.0)$ \\
\hline \multirow{2}{*}{\multicolumn{2}{|c|}{$\begin{array}{l}\text { Circumpapillary retinal nerve fiber layer thickness } \\
{[\mu \mathrm{m}]}\end{array}$}} & $69.8 \pm 12.3(46 \sim 100)$ \\
\hline & & \\
\hline OCT & Average & $\begin{array}{c}68.9 \pm 8.1(47 \sim 84) / 26.7 \pm 5.2(12 \sim 40) / 95.6 \pm \\
12.7(62 \sim 121)\end{array}$ \\
\hline GCIPL thickness $[\mu \mathrm{m}] /$ & Superotemporal & $71.2 \pm 10.4(38 \sim 90) / 32.7 \pm 8.6(10 \sim 47) / 103.9 \pm$ \\
\hline \multicolumn{2}{|l|}{ mRNFL thickness $[\mu \mathrm{m}] /$} & $17.4(60 \sim 132)$ \\
\hline
\end{tabular}


POAG: primary open angle glaucoma

NTG: normal tension glaucoma

LogMAR: Logarithm of the minimum angle of resolution

$\mathrm{mfPhNR} / \mathrm{B}$ : multifocal photopic negative response to multifocal B-wave ratio

$\mathrm{N} / \mathrm{T}$ : nasal to temporal amplitude ratio

HFA: Humphrey Field Analyzer

GCIPL: Ganglion cell-inner plexiform layer

mRNFL: Macular retinal nerve fiber layer

GCC: Ganglion cell complex

Values are mean \pm standard deviation (range)

Table 2 Correlation between the N/T of MfERG, the macula thickness of OCT, and the mean thresholds and the total deviation of HFA 10-2 in each sector in glaucoma patients 


\begin{tabular}{|c|c|c|c|c|c|}
\hline \multicolumn{2}{|l|}{ OCT and HFA } & \multicolumn{4}{|c|}{ vs N/T (IQR: 0.59-1.024) } \\
\hline Parameter & Sectors & Coefficient & 95\% LCL & 95\% UCL & $P$-value \\
\hline \multirow[t]{8}{*}{ GCIPL of OCT } & Average & -3.455 & -5.896 & -1.014 & 0.009 \\
\hline & Superotemporal & -4.767 & -7.592 & -1.942 & 0.003 \\
\hline & Superior & -3.461 & -6.021 & -0.901 & 0.014 \\
\hline & Superonasal & -5.311 & -8.19 & -2.433 & 0.001 \\
\hline & Inferonasal & -2.349 & -7.199 & 2.501 & 0.578 \\
\hline & Inferior & -2.134 & -6.211 & 1.943 & 0.522 \\
\hline & Inferotemporal & -3.027 & -6.023 & -0.032 & 0.032 \\
\hline & $\mathrm{SN}+\mathrm{IN} / \mathrm{ST}+\mathrm{IT}$ & -0.001 & -0.031 & 0.029 & 0.639 \\
\hline \multirow[t]{8}{*}{ mRNFL of OCT } & Average & -2.354 & -4.136 & -0.572 & 0.034 \\
\hline & Superotemporal & -3.665 & -6.407 & -0.923 & 0.026 \\
\hline & Superior & -4.455 & -7.321 & -1.589 & 0.008 \\
\hline & Superonasal & -2.546 & -4.118 & -0.973 & 0.007 \\
\hline & Inferonasal & 0.000 & -2.590 & 2.591 & 0.775 \\
\hline & Inferior & -1.182 & -4.261 & 1.897 & 0.624 \\
\hline & Inferotemporal & -2.706 & -5.414 & 0.002 & 0.132 \\
\hline & $\mathrm{SN}+\mathrm{IN} / \mathrm{ST}+\mathrm{IT}$ & 0.015 & -0.016 & 0.045 & 0.632 \\
\hline \multirow[t]{8}{*}{ GCC of OCT } & Average & -5.809 & -9.928 & $\begin{array}{l}-1.691 \\
\end{array}$ & 0.017 \\
\hline & Superotemporal & -8.432 & -13.613 & -3.252 & 0.007 \\
\hline & Superior & -7.916 & -13.221 & -2.61 & 0.008 \\
\hline & Superonasal & -7.857 & -12.041 & -3.673 & 0.001 \\
\hline & Inferonasal & -2.349 & -9.577 & 4.88 & 0.810 \\
\hline & Inferior & -3.316 & -10.332 & 3.7 & 0.642 \\
\hline & Inferotemporal & -5.733 & -10.939 & -0.527 & 0.055 \\
\hline & $\mathrm{SN}+\mathrm{IN} / \mathrm{ST}+\mathrm{IT}$ & 0.008 & -0.016 & 0.033 & 0.735 \\
\hline \multirow[t]{8}{*}{ Mean thresholds of HFA $10-2$} & Mean Deviation & -2.089 & -4.100 & -0.077 & 0.069 \\
\hline & Superotemporal & -1.759 & -4.173 & 0.656 & 0.231 \\
\hline & Superior & -4.302 & -7.772 & -0.833 & 0.045 \\
\hline & Superonasal & -4.437 & -7.652 & -1.223 & 0.020 \\
\hline & Inferonasal & 0.780 & -1.728 & 3.287 & 0.425 \\
\hline & Inferior & -0.606 & -2.360 & 1.149 & 0.773 \\
\hline & Inferotemporal & -0.864 & -2.433 & 0.706 & 0.026 \\
\hline & $\mathrm{SN}+\mathrm{IN} / \mathrm{ST}+\mathrm{IT}$ & 0.033 & -0.104 & 0.169 & 0.083 \\
\hline \multirow[t]{7}{*}{ Total deviation of HFA $10-2$} & Superotemporal & -1.511 & -3.588 & 0.567 & 0.097 \\
\hline & Superior & -4.329 & -7.861 & -0.797 & 0.051 \\
\hline & Superonasal & -4.823 & -8.172 & -1.475 & 0.018 \\
\hline & Inferonasal & 0.356 & -2.525 & 3.236 & 0.604 \\
\hline & Inferior & -0.282 & -1.932 & 1.369 & 0.943 \\
\hline & Inferotemporal & -0.219 & -1.012 & 0.573 & 0.569 \\
\hline & $\mathrm{SN}+\mathrm{IN} / \mathrm{ST}+\mathrm{IT}$ & -0.890 & -6.775 & 4.994 & 0.802 \\
\hline
\end{tabular}

Coefficient represents the increase in the value of parameter in each sector when N/T of mfERG changes by $75^{\text {th }}$ percentile from $25^{\text {th }}$ percentile. All multivariable regression models were adjusted for age and spherical equivalent.

$\mathrm{N} / \mathrm{T}$ : nasal to temporal amplitude ratio

mfERG: multifocal electroretinogram

OCT; Optical coherence tomopraphy 
HFA 10-2: Humphrey Field Analyzer Program Central 10-2

GCIPL₫Ganglion cell-inner plexiform layer

पRNFL: Macular retinal nerve fiber layer

GCC: Ganglion cell complex

IQR: interquartile range $\left(25^{\text {th }}\right.$ percentile $-75^{\text {th }}$ percentile)

LCL: lower confidence limit

UCL: upper confidence limit

Table 3 The $\mathrm{mfPhNR} / \mathrm{B}$ of $\mathrm{mfERG}$, the mean threshold and the total deviation of HFA 30-2, and the correlations between them in each sector in glaucoma patients

\begin{tabular}{|l|l|l|l|l|l|l|}
\hline HFA 30-2 & \multicolumn{5}{|c|}{ vs mfPhNR/B } \\
Parameter & Sectors & IQR & Coefficient & 95\% LCL & 95\% UCL & $P$-value \\
\hline Mean Threshold [dB] & Center & $0.269-0.385$ & -0.559 & -2.553 & 1.435 & 0.839 \\
& Superotemporal & $0.160-0.261$ & -1.632 & -3.028 & -0.235 & $\mathbf{0 . 0 1 3}$ \\
& Superonasal & $0.160-0.237$ & -0.271 & -2.654 & 2.112 & 0.684 \\
& Inferonasal & $0.193-0.299$ & 1.124 & -1.846 & 4.094 & 0.640 \\
& Inferotemporal & $0.194-0.317$ & 0.221 & -1.270 & 1.713 & 0.643 \\
\hline Total Deviation & Center & $0.269-0.385$ & -0.238 & -1.984 & 1.508 & 0.836 \\
& Superotemporal & $0.160-0.261$ & -1.701 & -3.145 & -0.258 & $\mathbf{0 . 0 1 8}$ \\
& Superonasal & $0.160-0.237$ & -0.368 & -2.755 & 2.019 & 0.720 \\
& Inferonasal & $0.193-0.299$ & 1.118 & -2.013 & 4.250 & 0.717 \\
& Inferotemporal & $0.194-0.317$ & 0.211 & -1.481 & 1.904 & 0.562
\end{tabular}

Coefficient in each sector represents the increase in the value of parameter of HFA 30-2 when $\mathrm{mfPhNR} / \mathrm{B}$ changes by $75^{\text {th }}$ percentile from $25^{\text {th }}$ percentile. All multivariable regression models were adjusted for age and spherical equivalent.

$\mathrm{mfPhNR} / \mathrm{B}$ : multifocal photopic negative response to multifocal B-wave ratio mfERG: multifocal electroretinogram

HFA 30-2: Humphrey Visual Field Analyzer Program Central 30-2

IQR: interquartile range $\left(25^{\text {th }}\right.$ percentile $-75^{\text {th }}$ percentile)

LCL: lower confidence limit

UCL: upper confidence limit 
Table 4 Correlations between the $\mathrm{mfPhNR} / \mathrm{B}$ of $\mathrm{mfERG}$ in each sector and the average thickness of the GCIPL, mRNFL, and GCC of OCT in glaucoma patients

\begin{tabular}{|c|c|c|c|c|c|}
\hline mfPhNR/B & \multicolumn{5}{|c|}{ vs GCIPL average } \\
\hline Sectors & IQR & Coefficient & | 95\% LCL & $95 \%$ UCL & $P$-value \\
\hline Center & $0.259-0.385$ & 0.839 & -1.456 & 3.133 & 0.739 \\
\hline Superotemporal & $0.151-0.261$ & -0.025 & -3.793 & 3.743 & 0.033 \\
\hline Superonasal & $0.160-0.240$ & 1.013 & -1.956 & 3.982 & 0.649 \\
\hline Inferonasal & $0.192-0.306$ & 1.096 & -1.831 & 4.024 & 0.726 \\
\hline Inferotemporal & 0.194-0..304 & 2.459 & -0.791 & 5.71 & 0.042 \\
\hline & \multicolumn{5}{|c|}{ vs mRNFL average } \\
\hline Center & $0.259-0.385$ & 0.863 & -0.720 & 2.446 & 0.551 \\
\hline Superotemporal & $0.151-0.261$ & 1.04 & -1.443 & 3.523 & 0.228 \\
\hline Superonasal & $0.160-0.240$ & 1.283 & \begin{tabular}{|l|}
-0.919 \\
\end{tabular} & 3.485 & 0.483 \\
\hline Inferonasal & $0.192-0.306$ & 1.492 & -0.414 & 3.399 & 0.301 \\
\hline Inferotemporal & $0.194-0 . .304$ & 2.363 & 0.416 & 4.31 & 0.003 \\
\hline & \multicolumn{5}{|c|}{ vs GCC average } \\
\hline Center & $0.259-0.385$ & 1.701 & -2.049 & 5.451 & 0.650 \\
\hline Superotemporal & $0.151-0.261$ & 1.015 & -5.103 & 7.134 & 0.061 \\
\hline Superonasal & $0.160-0.240$ & 2.296 & -2.778 & 7.370 & 0.642 \\
\hline Inferonasal & $0.192-0.306$ & 2.588 & -2.076 & 7.253 & 0.540 \\
\hline Inferotemporal & $0.194-0 . .304$ & 4.823 & -0.234 & 9.88 & 0.012 \\
\hline
\end{tabular}

Coefficient represents the increase in the value of $\mathrm{mfPhNR} / \mathrm{B}$ in each sector when GCIPL average, mRNFL average or GCC average changes by $75^{\text {th }}$ percentile from $25^{\text {th }}$ percentile. All multivariable regression models were adjusted for age and spherical equivalent.

$\mathrm{mfPhNR} / \mathrm{B}$ : multifocal photopic negative response to multifocal B-wave ratio mfERG: multifocal electroretinogram

OCT; Optical coherence tomopraphy

GCIPL $\square$ Ganglion cell-inner plexiform layer

mRNFL: Macular retinal nerve fiber layer

GCC: Ganglion cell complex

LCL: lower confidence limit

UCL: upper confidence limit

\section{Figures}




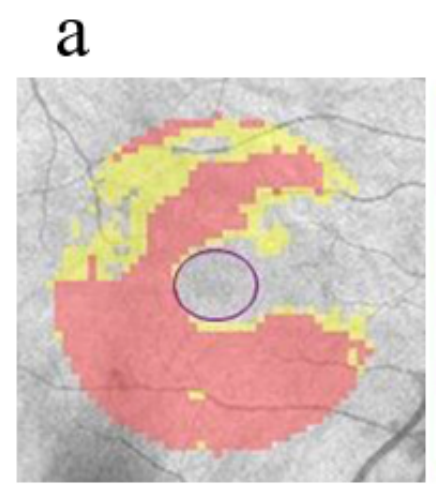

Deviation Map

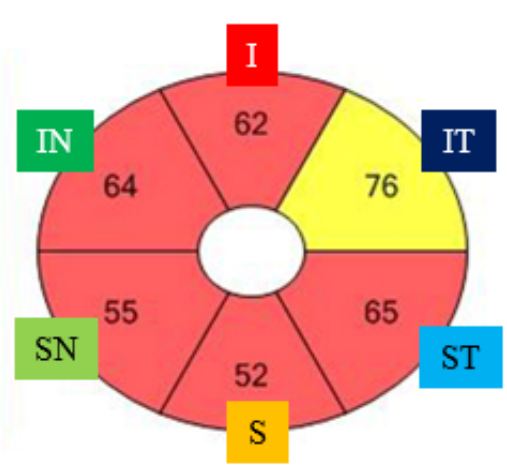

sectors

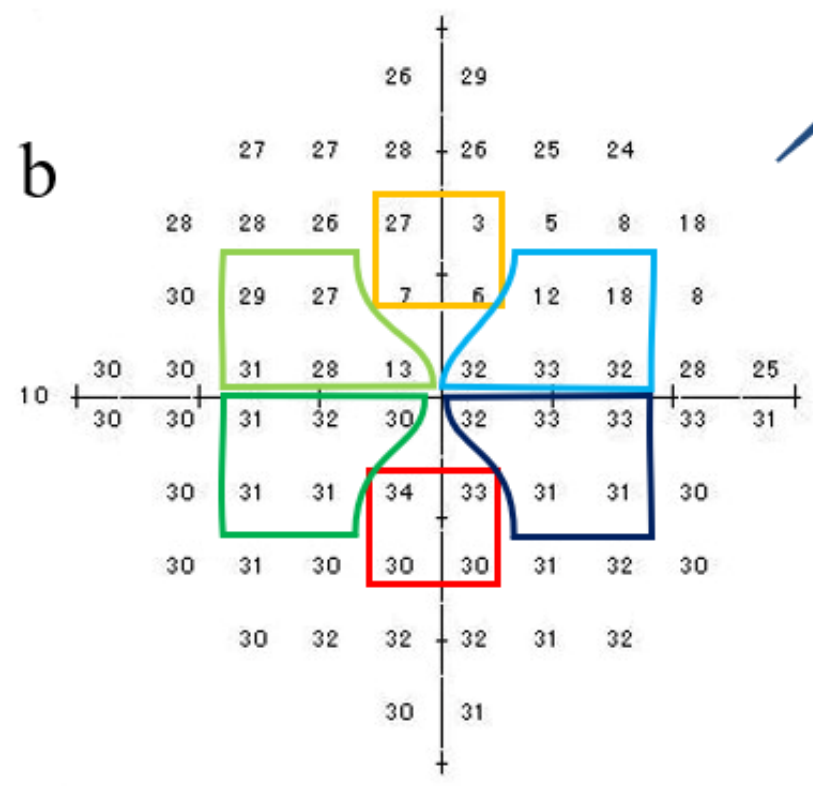

Right eye

CENTFEL 10-2 THIRSFOLD TES

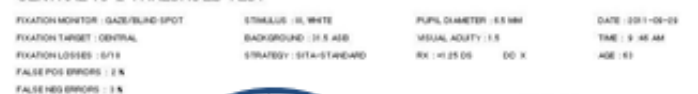
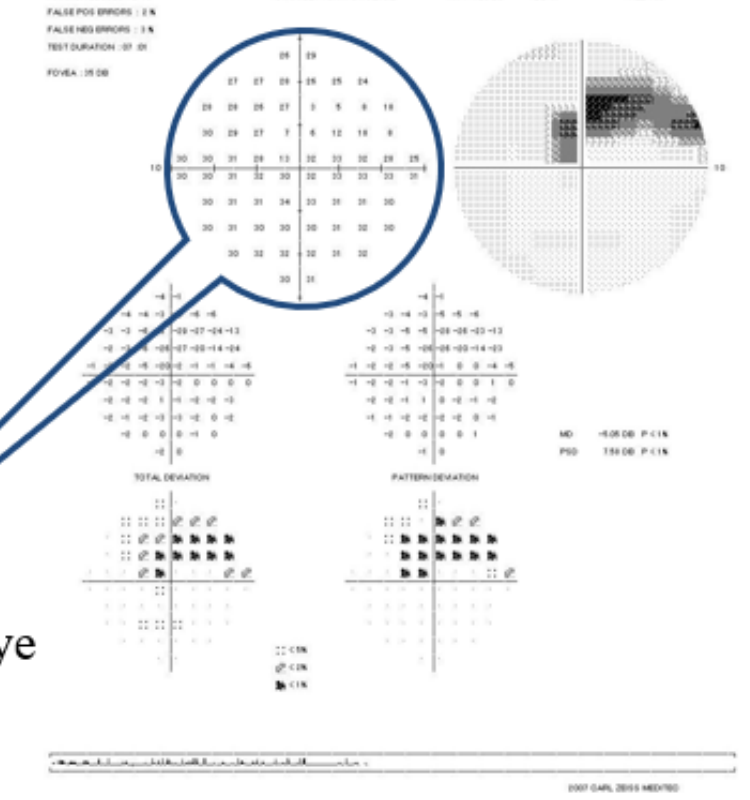

\section{Superior (S)}

Superotemporal (ST)

$\square$ Inferotemporal (IT)

$\square$ Inferior (I)

Superonasal (SN)

$\square$ Inferonasal (IN)

\section{Figure 1}

Association between optical coherence tomography (OCT) thickness and Humphrey Field Analyzer Central 10-2 program (HFA10-2) (a). The macular thickness is measured by OCT between two concentric circles of 2- and 6-mm diameters. (b) The findings of the HFA10-2 in right eye of glaucoma patient. Based on the retinal ganglion cell displacement [16], we classified the stimulus points on the HFA10-2 corresponding to the six sectors of the ganglion cell and inner plexiform layer (GCIPL) measurement ellipse into 6 groups (i.e., superior, superotemporal, inferotemporal, inferior, superonasal, and inferonasal sectors). 


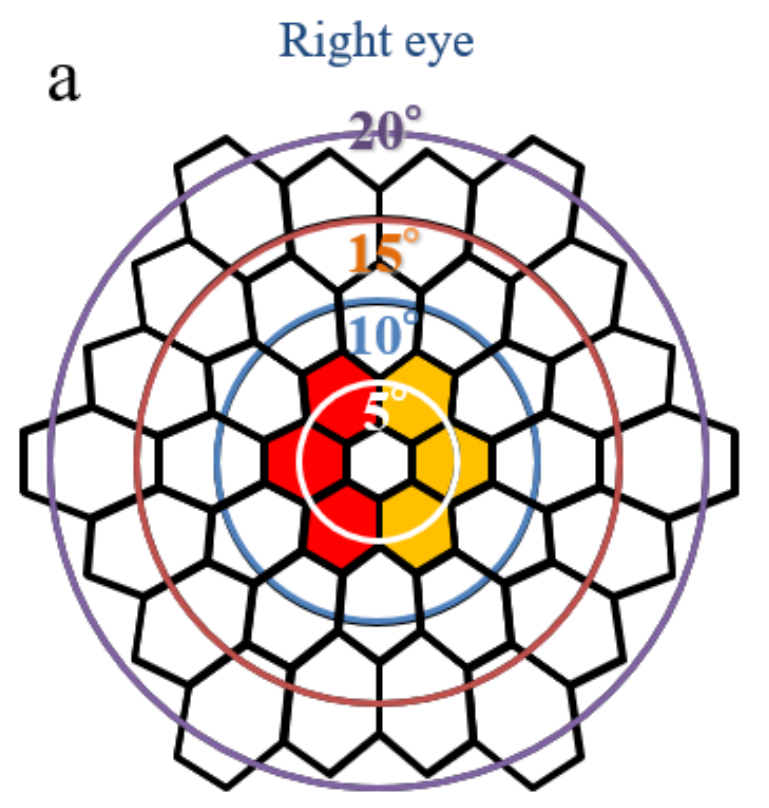

C

Right eye

Nasal Whe when Nhen New Temporal

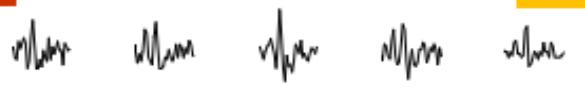
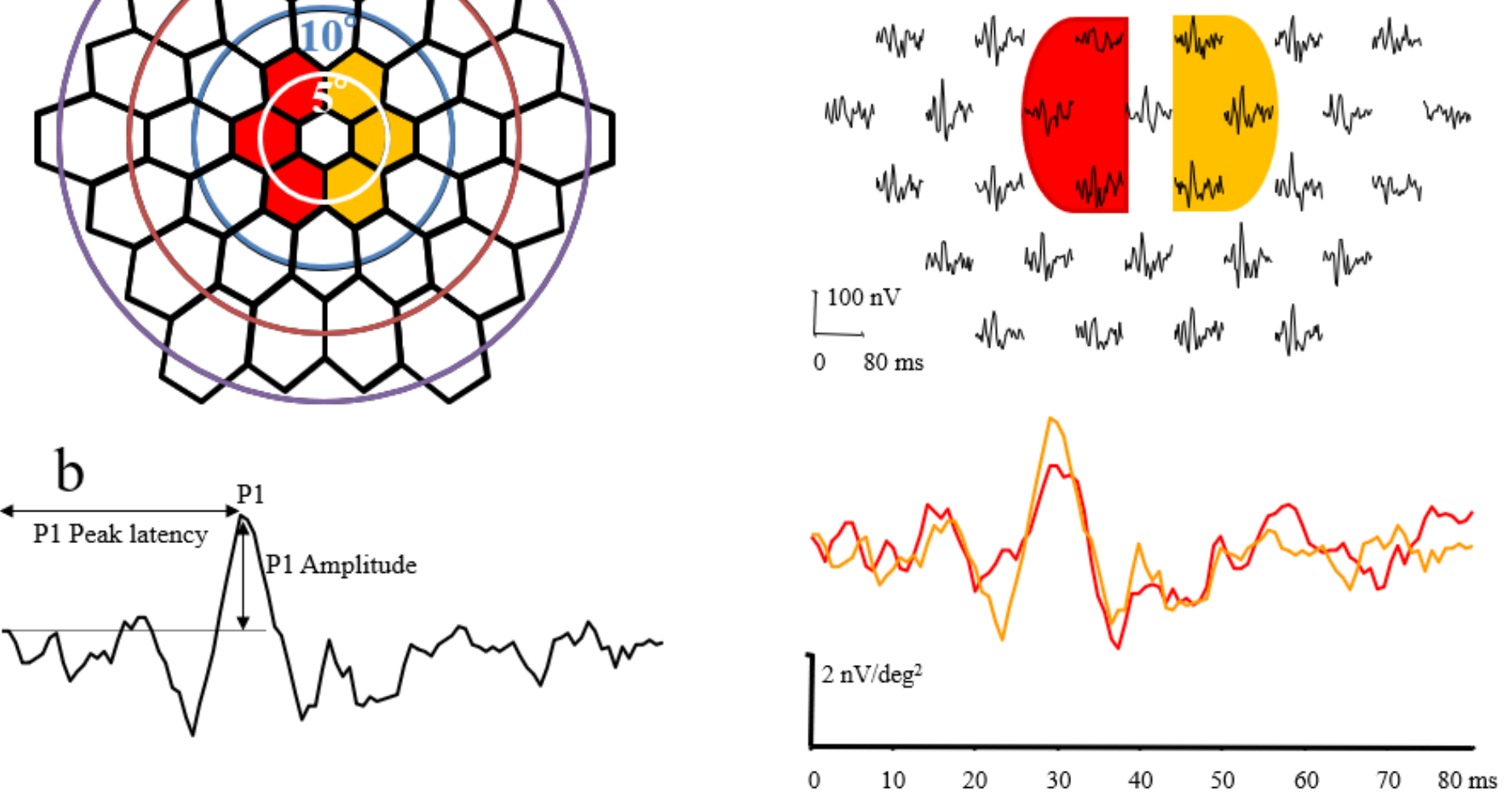

Figure 2

The stimulus array and the nasal to temporal amplitudes ratio obtained from multifocal electroretinograms (mfERGs) (a). The pattern of the 37-hexagon stimulus array with circles indicating radii of $5^{\circ}, 10^{\circ}, 15^{\circ}$ and $20^{\circ}$ in right eye. (b). The measurements of the first slice of the second-order kernels obtained from multifocal electroretinograms (mfERGs). The first positive peak, P1 amplitude was measured. (c). We separated the hexagons and averaged them according to the temporal (orange color) nasal (red color) hemispheres. We used the ratio of the amplitudes of the mfERGs of the nasal to the temporal hemisphere within the central $5^{\circ}(\mathrm{N} / \mathrm{T})$ to evaluate the asymmetry. 

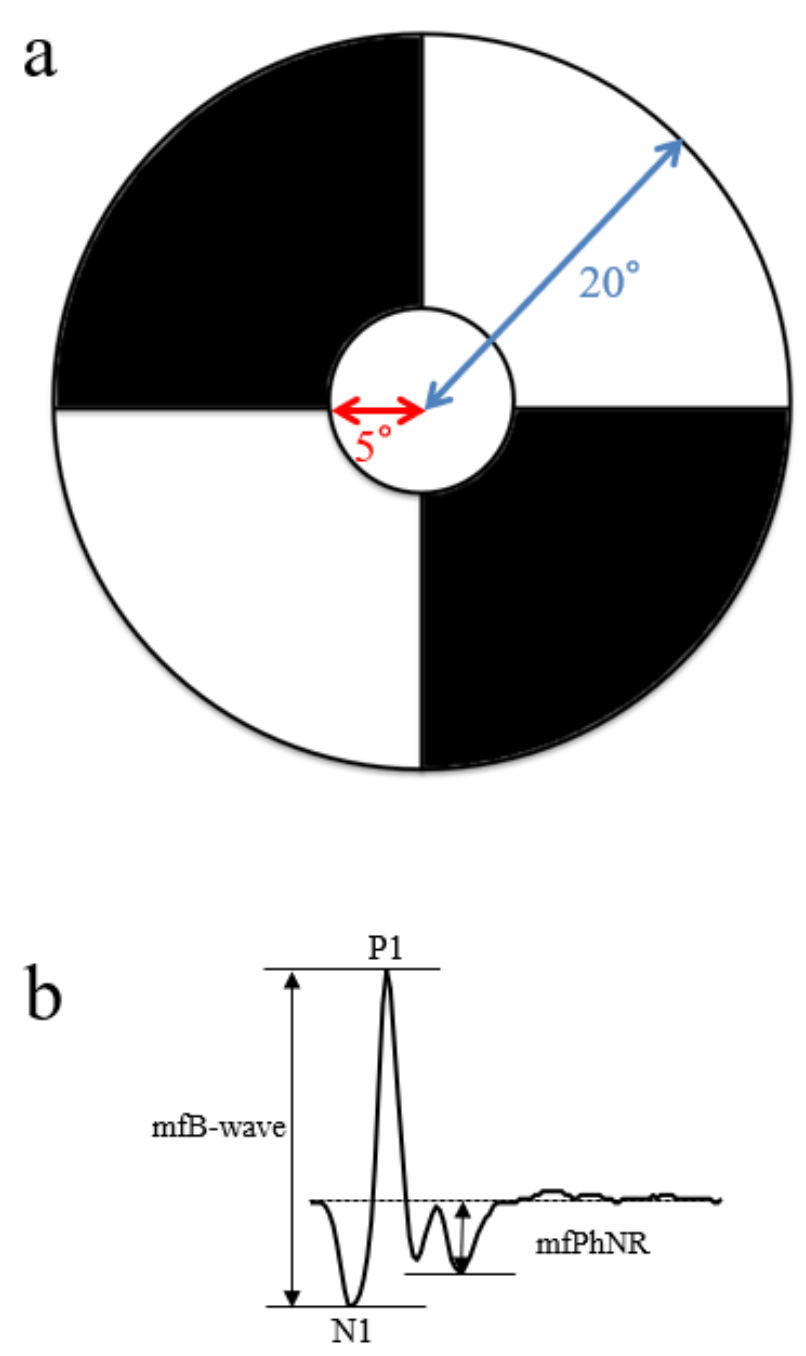

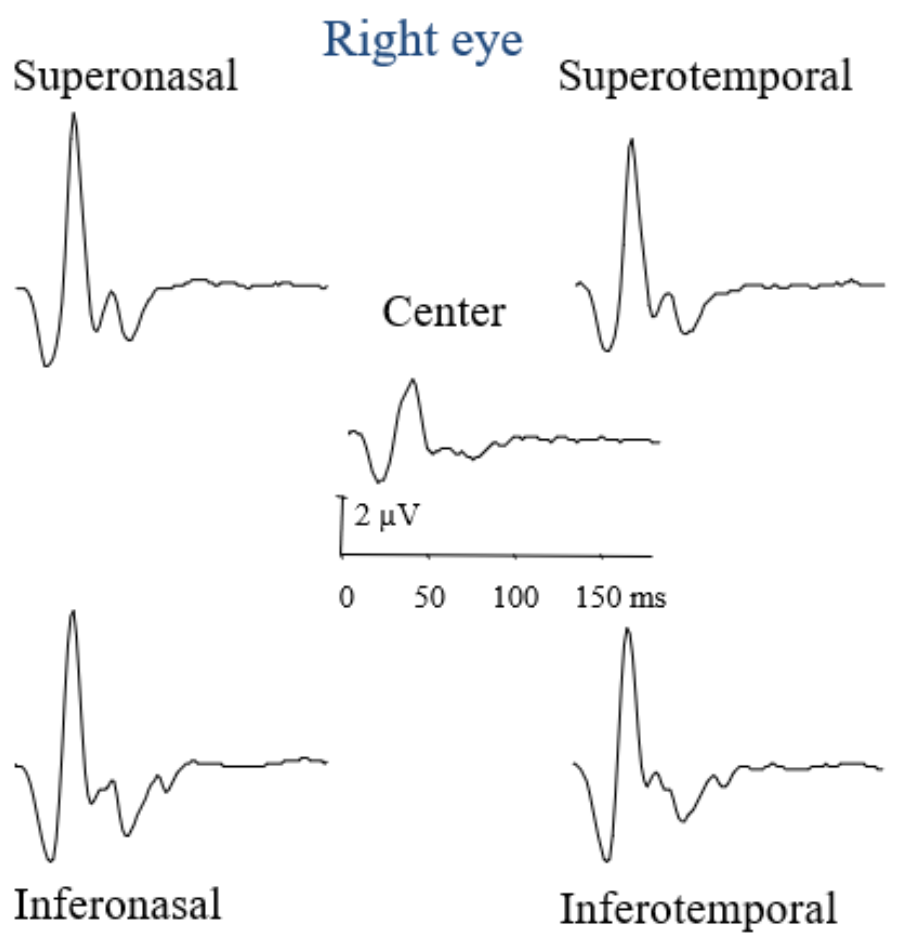

\section{Figure 3}

The stimulus patterns and the multifocal photopic negative response (mfPhNR) (a). The stimulus patterns ( a circular stimulus with a $5^{\circ}$ radius centered on the fovea and a quarter of an annulus placed in the superotemporal, superonasal, inferotemporal, and inferonasal regions around the fovea) were used to elicit mfERGs. The radius of the inner border of the annulus was $5^{\circ}$ and that of the outer border was $20^{\circ}$. (b). The representative waveforms of the mfERGs recoded from five sectors in right eye of a patient with glaucoma. We measured the multifocal B-wave (mfB-wave; P1-N1) from the first negative trough to the peak of the following positive wave. The mfPhNR was measured from the baseline to the negative trough at more than $70 \mathrm{~ms}$ from the stimulus onset. 


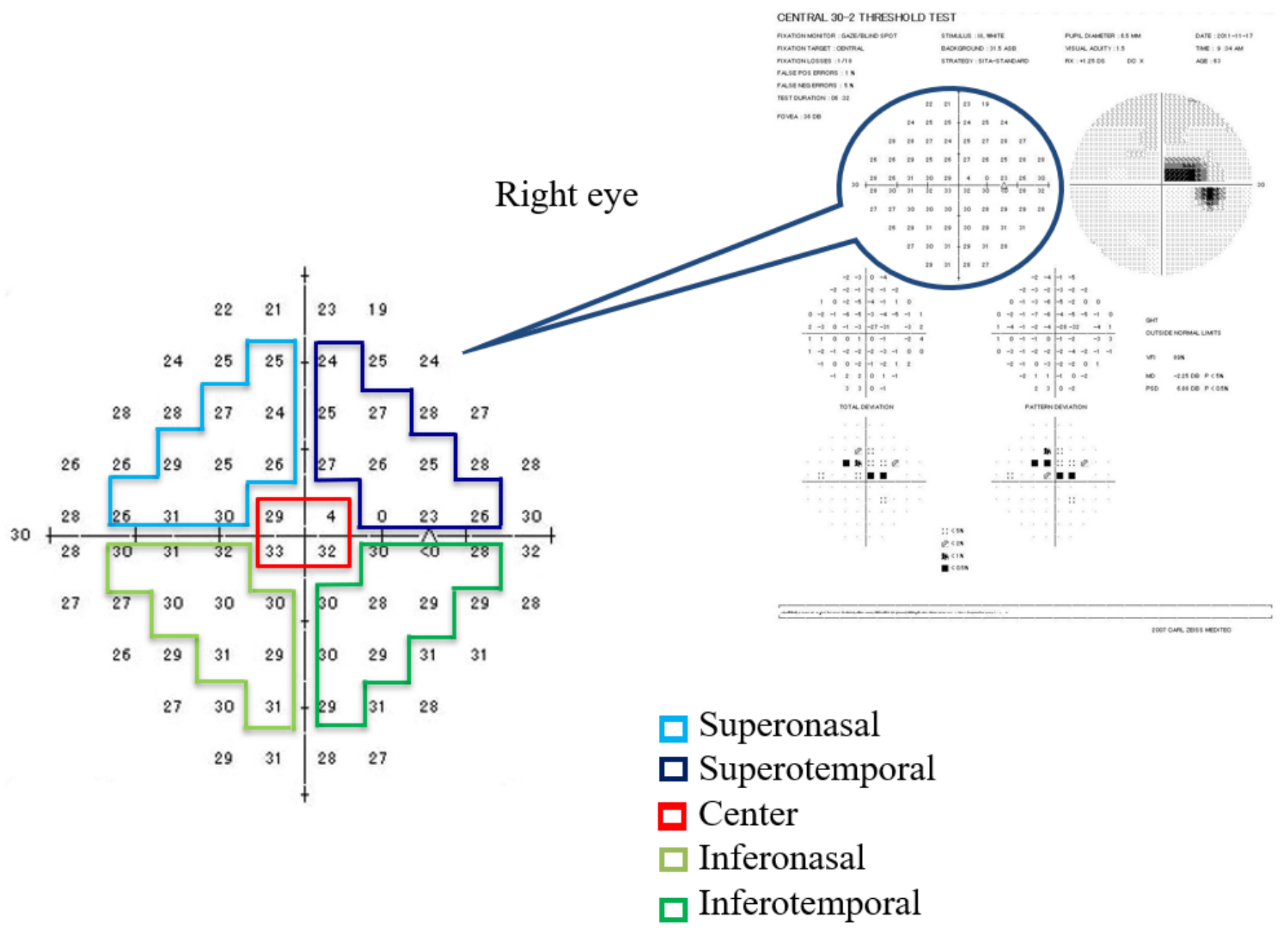

Figure 4

Humphrey Field Analyzer Central 30-2 program (HFA 30-2) corresponding to the mfPhNR regions. (see Figure 3). To compare the multifocal photopic negative response to B-wave ratio ( $\mathrm{mfPhNR} / \mathrm{B})$ with the corresponding visual field findings, we measured the thresholds with the HFA 30-2 and averaged for the same sectors (i.e., superonasal, superotemporal, center, inferonasal, and inferotemporal) according to the distance from the macula within the central $20^{\circ}$.

\section{Supplementary Files}

This is a list of supplementary files associated with this preprint. Click to download.

- Fig5.SupplementalFiguresforTabale2Tanaka2020.pptx

- Fig6.SupplementalFiguresForTabale3Tanaka2020.pptx

- Fig7.SupplemetnalFiguresforTable4Tanaka2020.pptx 